\title{
Nested codes for constrained memory and for dirty paper
}

\author{
Hans Georg Schaathun ${ }^{1}$ and Gérard D. Cohen ${ }^{2}$ \\ 1 Dept. Informatics, \\ University of Bergen \\ Pb. 7800 \\ N-5020 Bergen \\ Norway \\ 2 Dept. Informatique et Reseaux, \\ Ecole Nationale Supérieure des Télécommunications \\ 46, rue Barrault, \\ F-75634 Paris Cedex 13 \\ France
}

\begin{abstract}
Dirty paper coding are relevant for wireless networks, multiuser channels, and digital watermarking. We show that the problem of dirty paper is essentially equivalent to some classes of constrained memories, and we explore the binary so-called nested codes, which are used for efficient coding and error-correction on such channels and memories.
\end{abstract}

Keywords: dirty paper, constrained memory, nested codes, covering codes

The motivation of this paper is the dirty paper channel introduced by Costa [3]. This channel has received increased attention [4] in recent years, due to applications in wireless multiuser networks and digital fingerprinting [5].

We show that the dirty paper channel is practically equivalent to writing on reluctant memories, and we make a few improvements on the existing results for such channels. Our interest is mainly in the binary dirty paper channel (BDP).

\section{Dirty paper and constrained memory coding}

The dirty paper channel is depicted in Figure 1. There are two independent noise sources which are added to the transmitted signal to form the received signal. The first noise vector, which we will call the state of the channel is known to the sender but not to the receiver. The second noise vector, which we will refer to as noise is unknown to both.

The sender is subject to a power constraint $\|\mathbf{x}\| \leq P$ on the transmitted signal. For a binary channel $\|\cdot\|$ is usually the Hamming norm; for a continuous channel it is usually the Euclidean norm.

Costa [3] introduced this channel with Gaussian sources for both the state and the noise. His surprising result was that the channel capacity depends only on the intensity of the noise; the intensity of the state does not change capacity. In 


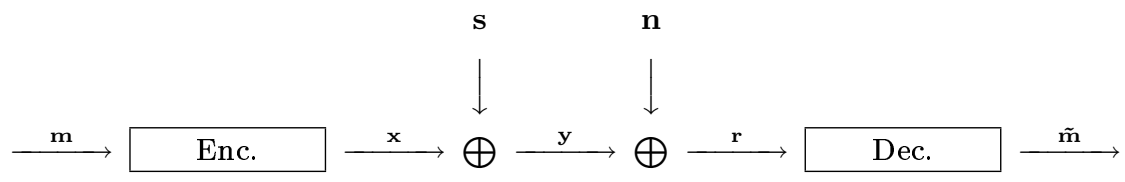

Figure 1. The dirty paper channel.

more recent years, his results have been generalised to other source distributions. We will consider the binary dirty paper channel (BDP).

In a constrained memory, there are restrictions on writing to the memory, such that starting in one memory state, some states are reachable in one write operation and others are not. For each memory state, there is a feasible region of words which may be used to represent the next message. In this case the state is given by the previous message stored in memory.

Dirty paper coding and constrained memory coding are similar, in fact BDP channels are practically equivalent to WRM (write reluctant memories) with error-correction [2]. In WRM, one write operation cannot change more than a certain number $P$ of bits. This corresponds to the power constraint in BDP; if $\mathbf{s}$ is the state (previous contents), $\mathbf{x}$ is the change, and $\mathbf{y}=\mathbf{s}+\mathbf{x}$ is the memory contents after writing, then $w(\mathbf{x}) \leq P$.

The state on dirty paper channels is externally given, whereas in constrained memories it is the old codeword (with possible errors). The state, together with power constraints, defines the feasible region of vectors $\mathbf{y}$ which can be generated. For BDP/WRM, the feasible region is a Hamming sphere around the state.

Remark 1. Occasionnally, in constrained memories, one assumes that $\mathbf{s}$ is a codeword with few errors, since nobody would write rubbish to the memory. We will not make this assumption, for two reasons. Primarily, it does not extend to BDP. Also, we know of no cases where results can be improved due to this assumption. Furthermore, by avoiding such assumption, the system can recover after an error pattern which could not be corrected.

Example 1. Another example of constrained memory is the Write Isolated Memory (WIM), where two consecutive memory bits cannot be changed in the same operation. In other words, the feasible region is the set $\left\{\mathbf{x}+\mathbf{s}: \mathbf{x}=\left(x_{1}, \ldots, x_{n}\right), x_{i}=\right.$ $\left.1 \Rightarrow x_{i-1}=x_{i+1}=0\right\}$, where $\mathbf{s}$ is the memory state and $x_{0}=x_{n+1}=0$ by convention.

BDP (WRM) and WIM both fall into a class of channels, where the feasible regions are translation invariant, permitting some common techniques. By this we mean that if $F_{\mathbf{s}}$ is the feasible region from $\mathbf{s}$, then $F_{\mathbf{s}^{\prime}}=F_{\mathbf{s}}-\mathbf{s}+\mathbf{s}^{\prime}$. Let us call this class CCTIR (constrained channels with translation invariant regions). 


\section{Some coding theory}

An $(n, M)_{q}$ code $C$ is an $M$-set of $n$-tuples over a $q$-ary alphabet. When $q=2$ we may suppress the subscript. The Hamming distance $d(\mathbf{x}, \mathbf{y})$ is the number of positions where the two tuples differ. The minimum distance $d=d(C)$ of $C$ is the least distance between two different codewords. We say that $C$ is an $(n, M, d)_{q}$ code. The covering radius $r$ of $C$ is the largest distance between a vector $\mathbf{y} \in Q^{n}$ and the code.

The problem of covering codes amounts to finding codes minimising $r$ given $n$ and $M$, whereas the problem of error-correcting codes is about maximising $d$ given $n$ and $M$.

We also define normalised measures, which will be useful when moving to asymptotic codes. We define the rate $\log _{q} M / n$, the distance $\delta=d / n$, and the covering radius $\rho=r / n$.

\section{Codes for CCTIR}

In order to make a successful code for CCTIR, we need for every state $\mathbf{s}$ and every message $\mathbf{m}$, to have at least one codeword $\mathbf{x}$ corresponding to $\mathbf{m}$ in the feasible region of $\mathbf{s}$. Furthermore, we require any capability for error-correction that we may need. We will study e-error correcting CCTIR codes.

Lemma 1. For CCTIR, if $\mathrm{x} \in F_{\mathbf{y}}$ then $\mathbf{y} \in F_{\mathbf{x}}$.

Let $B_{i}$ be the set of words corresponding to message $i$. We require that for any $\mathbf{s}, F_{\mathbf{s}} \cap B_{i} \neq \emptyset$. By the lemma above, this is equivalent to

$$
\bigcup_{\mathbf{b} \in B_{i}} F_{\mathbf{b}}=\mathbb{F}^{n}
$$

i.e. that the feasible regions around the words of $B_{i}$ cover the space. If the set of possible messages is $i=1, \ldots, M$, then we define

$$
C_{F}=\bigcup_{i=1}^{M} B_{i} .
$$

When the feasible regions are spheres of radius $\rho$, this is to say that $B_{i}$ must be a covering code of covering radius $\rho$ or smaller. For other feasible regions it is a more general covering by F-shapes.

In order to correct up to $e$ errors, we require that if $i \neq j$, then $d\left(B_{i}, B_{j}\right)>2 e$. It is sufficient to require that $C_{F}$ has minimum distance $2 e+1$ or more; i.e. that $C_{F}$ is $e$-error correcting. Furthermore as a necessary condition, if there are two codewords with distance at most $2 e$ apart, they must fall in the same set $B_{i}$.

In a sense, we try to pack the space with coverings $B_{i}$ such that we maintain a minimum distance of $2 e+1$, a problem studied in [2]. 
We say that a CCTIR code $\left(B_{1}, \ldots, B_{M}\right)$ is linear if $C_{F}$ is a linear $e$-errorcorrecting code, $B_{j}$ is a subcode satisfying (1) for some $j$, and the $B_{i}$ are cosets of $B_{j}$ in $C_{F}$. Clearly by linearity, $B_{i}$ satisfies (1) whenever $B_{j}$ does.

Let $a_{n}=\# F_{\mathbf{0}}$. For CCTIR, all the feasible regions clearly have the same size.

Lemma 2. For an $(n, M)$ CCTIR code, we have

$$
M \leq a_{n}
$$

Lemma 3. For dirty paper codes, we have

$$
a_{n}=V(n, R)=\sum_{i=0}^{n}\left(\begin{array}{c}
n \\
i
\end{array}\right) .
$$

In the case of WRM and dirty paper channels, a linear CCTIR code is also called a nested code. We call $C_{F}$ the fine code and $C_{C} \subseteq C_{F}$ the coarse code. The nested code is the quotient $C=C_{F} / C_{C}$, and we say that $C$ is an $\left[n, K ; d_{1}, \rho\right]$ code, where $K=k_{F}-k_{C}$ is the dimension of $C$. The following lemma is well known.

Lemma 4 (Supercode lemma). For any $\left[n, K ; d_{1}, \rho\right]$ nested code, we have $\rho \geq d_{1}$.

\section{Asymptotic existence}

Definition 1 (Entropy). The (binary) entropy of a discrete stochastic variable $X$ drawn from a set $\mathcal{X}$ is defined as

$$
H(X)=-\sum_{x \in \mathcal{X}} P(X=x) \log P(X=x) .
$$

The conditional entropy of $X$ with respect to another discrete stochastic variable $Y$ from $\mathcal{Y}$ is

$$
H(X \mid Y)=-\sum_{y \in \mathcal{Y}} P(Y=y) \sum_{x \in \mathcal{X}} P(X=x \mid Y=y) \log P(X=x \mid Y=y) .
$$

The following general theorem appears in [2].

Theorem 1. For $n$ large enough, there are $\theta n$-error correcting codes for CCTIR with rate

$$
\kappa(\theta) \geq \kappa_{0}-H(2 \theta),
$$

where $\kappa_{0}$ is the maximum rate for a non-error-correcting code for the same constrained channel.

The proof is by greedy techniques, as follows. 
Proof. We write

$$
S(B, i)=\bigcup_{\mathbf{b} \in B}\{\mathbf{x}: d(\mathbf{x}, \mathbf{b} \leq i\} .
$$

First we make a code $C_{C}$ of rate $1-\kappa$ without error-correction. Let $S_{0}=$ $S\left(C_{C}, 2 \theta n-1\right)$.

We start with $B=\{\mathbf{0}\}$, and construct a code $C_{C}$ by the following greedy algorithm. In each step we take a random vector $\mathbf{y} \in S \backslash S\left(B+C_{C}, 2 \theta n-1\right)$, and update $B$ to be the linear span of $\mathbf{y}$ and the vectors of $B$. We proceed until $S \backslash S\left(B+C_{C}, 2 \theta n-1\right)$ is empty. Since each word included in $B$ excludes at most $\# S\left(C_{C}, 2 \theta n-1\right)$ elements from $S_{0}$, we get that

$$
\# B \geq \frac{2^{n}}{\# C_{C} \# S(\{\mathbf{0}\}, 2 \theta n-1)} \geq \frac{2^{\kappa n}}{\# S(\{\mathbf{0}\}, 2 \theta n-1)} .
$$

Assymptotically, we have $\# B \approx 2^{(\kappa-H(2 \theta)) n}$, Let $C_{F}=B+C_{C}$, so that $C=$ $C_{F} / C_{C} \equiv B$. Clearly the rate of $B$ and $C$ is $\kappa-H(2 \theta)$ as required.

In the case of dirty paper channel, $\kappa_{0}=1-R_{\rho}$ where $R_{\rho}$ is the minimum rate for a covering code with appropriate $\rho$.

Theorem 2. For dirty paper codes with no error-correction, we can obtain rate $\kappa_{0}=H(\rho)$.

Observe that whenever $\rho>\delta$, we get asymptotic codes with non-zero rate from the above theorems. For $\rho=\delta$, however, the guaranteed rate is just zero.

Problem 1 Are there asymptotic families of nested codes with $R>0$ and $\rho=d$ ?

\section{Some small constructions}

\begin{tabular}{|c|c|c|}
\hline Parameters & Coarse code & Fine code \\
\hline \hline$[3,1 ; 1,1]$ & {$\left[\begin{array}{l}110 \\
101\end{array}\right]$} & {$\left[\begin{array}{l}100 \\
010 \\
001\end{array}\right]$} \\
\hline$[3,1 ; 2,2]$ & {$[110]$} & {$\left[\begin{array}{l}110 \\
011\end{array}\right]$} \\
\hline$[3,2 ; 1,2]$ & {$[110]$} & {$\left[\begin{array}{l}100 \\
010 \\
001\end{array}\right]$} \\
\hline
\end{tabular}

Table 1. Some nested codes for $n=3$.

Lemma 5. For any $[n, K ; 1,1]$ nested code with even $n$, we have $K \leq \log n$. 


\begin{tabular}{|c|c|c|}
\hline Parameters & Coarse code & Fine code \\
\hline \hline$[4,2 ; 1,1]$ & {$\left[\begin{array}{l}1110 \\
1001\end{array}\right]$} & {$\left[\begin{array}{l}1000 \\
0100 \\
0010 \\
0001\end{array}\right]$} \\
\hline$[4,2 ; 2,2]$ & {$[1111]$} & {$\left[\begin{array}{l}1100 \\
1010 \\
1001\end{array}\right]$} \\
\hline$[4,3 ; 1,2]$ & {$[1111]$} & {$\left[\begin{array}{l}1000 \\
0100 \\
0010 \\
0001\end{array}\right]$} \\
\hline \hline$[5,2 ; 1,1]$ & {$\left[\begin{array}{l}11100] \\
10011 \\
00110\end{array}\right]$} & {$[5,5,1]$ full code } \\
\hline$[5,4 ; 1,2]$ & {$[11111]$} & {$[5,5,1]$ full code } \\
\hline$[5,2 ; 2,2]$ & {$[11111]$} & {$\left[\begin{array}{l}11000 \\
10100 \\
10010\end{array}\right]$} \\
\hline$[6,2 ; 2,2]$ & {$\left[\begin{array}{l}111000 \\
000111\end{array}\right]$} & {$\left[\begin{array}{l}111000 \\
000111 \\
100100 \\
010010\end{array}\right]$} \\
\hline$[6,4 ; 1,2]$ & {$\left[\begin{array}{l}111000 \\
000111\end{array}\right]$} & {$[6,6,1]$ full code } \\
\hline$[6,4 ; 2,3]$ & {$[111111]$} & {$[6,5,2]$ even weight } \\
\hline
\end{tabular}

Table 2. Some nested codes for $n=4,5,6$. 
Proof. For a $\left[n, k_{C}\right] 1$ covering code, we have $k_{C} \leq n-\log n$ when $n$ is even, and for an $\left[n, k_{F}, 1\right]$ code, we have $k_{F} \leq n$. Hence $K=k_{F}-k_{C} \leq \log n$.

Lemma 6. There is a $\left[2^{K}-1, K ; 1,1\right]$ nested code for any $K$.

Proof. Let the coarse code be the $\left[2^{K}-1,2^{K}-1-K, 3\right] 1$ Hamming code, and let the fine code be the $\left[2^{K}-1,2^{K}-1,1\right]$ code.

Lemma 7. There are $\left[2^{K}, K ; 1,1\right]$ and $\left[2^{K}-1, K ; 1,1\right]$ nested codes.

Proof. The fine code is the $[n, n, 1]$ full space. The coarse codes are the Hamming codes, and the $\left[2^{K}, 2^{K}-K, 1\right]$ direct sum of a Hamming code padded with a zero column, and the code generated by a single word of weight one.

Lemma 8. There are $\left[2^{K}, K ; 2^{K-1}, 2^{K-1}\right]$ and $\left[2^{K}-1, K ; 2^{K-1}-1,2^{K-1}-1\right]$ nested codes for any positive $K$.

Proof. Let the coarse code be the $\left[2^{K}, 1,2^{K}\right]$ repetition code, and let the fine code be the $\left[2^{K}, K, 2^{K-1}\right]$ Reed-Muller code. The second set of parameters comes from puncturing the above code.

Lemma 9. If there is an $[n, K ; d, \rho]$ code, then there is an $[n-1, K ; d-1, \rho]$ code by puncturing and an $[n-1, K ; d, \rho+1]$ code by shortening.

Proof. This follows easily from the standard results on puncturing and shortening of error-correcting and covering codes.

Lemma 10 ([2]). The $\left[2^{m}-1,2^{m}-1-2 m, 5\right]$ BCH code has $\rho=3$ for $m \geq 3$.

Corollary 1. There is a $\left[2^{m}-1, m ; 3,3\right]$ nested code for every $m \geq 3$.

Proof. The coarse code is the $\left[2^{m}-1,2^{m}-1-2 m, 5\right] 3 \mathrm{BCH}(2)$ code, and the fine code is the Hamming code.

Lemma 11 ([2]). The $\left[2^{m}-1,2^{m}-1-3 m, 7\right]$ BCH code has $\rho=5$ for $m \geq 4$.

Corollary 2. There is a $\left[2^{m}-1, m ; 5,5\right]$ nested code for every $m \geq 4$.

Proof. The coarse code is the $\left[2^{m}-1,2^{m}-1-3 m, 7\right] 5 \mathrm{BCH}(3)$ code, and the fine code is the $\left[2^{m}-1,2^{m}-1-2 m, 5\right] 3 \mathrm{BCH}(2)$ code.

Lemma 12. There are $\left[2^{2 m+1}-2^{m}, 2 m+2 ; 2^{2 m}-2^{m}, 2^{2 m}\right]$ and $\left[2^{2 m+1}-2^{m}-\right.$ $\left.1,2 m+2 ; 2^{2 m}-2^{m}-1,2^{2 m}-1\right]$ nested codes

Proof. The coarse code is a repetition code. The fine code is a $\left[2^{2 m+1}-2^{m}, 2 m+\right.$ $3,2^{2 m}-2^{m}$ ] code [1] or a punctured version of it.

Lemma 13. There is no $[6,4 ; 2,2]$ nested code, so the $[6,3 ; 2,2],[6,4 ; 1,2]$ and $[6,4 ; 2,3]$ codes are optimal.

Proof. The smallest covering code of $\rho=2$ and $n=6$ has $k_{C}=2$, so to get $K=4$, we would need $k_{F} \geq 6$, which would give $d=1$. 


\begin{tabular}{|c|c|c|}
\hline Parameters & Coarse code & Fine code \\
\hline$\overline{[\overline{[7,3 ; 1,1]}}$ & {$[7,4 ; 3] 1$ Hamming } & $\overline{[7,7,1]}$ \\
\hline$[7,3 ; 3,3]$ & {$[7,1,7] 3$ repetition } & {$[7,4,3]$ Hamming } \\
\hline$[8,3 ; 1,1]$ & {$\left[\begin{array}{l}10001110 \\
01000110 \\
00101010 \\
00010110 \\
00000001\end{array}\right]$} & {$[8,8,1]$} \\
\hline$[8,3 ; 4,4]$ & {$[8,1,8] 4$ repetition } & {$[8,4,4]$ ext. Hamming } \\
\hline$[15,4 ; 3,3]$ & {$[15,7,5] 3 \mathrm{BCH}(2)$} & {$[15,11,3]$ Hamming } \\
\hline$[15,2 ; 5,5]$ & {$[15,5,7] 5 \mathrm{BCH}(3)$} & {$[15,7,5] 3 \mathrm{BCH}(2)$} \\
\hline$[15,2 ; 7,7]$ & {$[15,1,15] 7$ repetition } & {$[15,3,7] \mathrm{BCH}(3)$} \\
\hline$[15,4 ; 7,7]$ & {$[15,1,15] 7$ repetition } & {$[15,5,7]$ punctured Reed-Muller } \\
\hline$[16,6 ; 4,6]$ & {$[16,5,8] 6 \mathrm{RM}(1,4)$} & {$[16,11,4] \operatorname{RM}(2,4)$} \\
\hline$[16,4 ; 8,8]$ & {$[16,1,16] 8$ repetition } & {$[16,5,8]$ Reed-Muller } \\
\hline$[27,6 ; 11,13]$ & {$[27,1,27] 13$ repetition } & {$[27,7,11][1]$} \\
\hline$[28,6 ; 12,14]$ & {$[28,1,28] 14$ repetition } & {$[28,7,12][1]$} \\
\hline$[31,5 ; 3,3]$ & {$[31,21,5] 3 \mathrm{BCH}(2)$} & $31,26,3]$ Hamming \\
\hline$[31,5 ; 5,5]$ & {$[31,16,7] 5 \mathrm{BCH}(3)$} & {$[31,21,5] 3 \mathrm{BCH}(2)$} \\
\hline$[31,5 ; 7,7]$ & {$[31,11,11] 7 \mathrm{BCH}(4)$} & {$[31,16,7] 5 \mathrm{BCH}(3)$} \\
\hline$[31,5 ; 11,11]$ & {$[31,6,15] 11 \mathrm{BCH}(6)$} & $31,11,11] 7 \mathrm{BCH}(4)$ \\
\hline$[31,5 ; 15,15]$ & {$[31,1,31] 15$ repetition } & {$[31,6,15]$ punctured Reed-Muller } \\
\hline$[32,5 ; 2,2]$ & {$[32,26,4] 2 \mathrm{RM}(3,5)$} & {$[32,31,2] \mathrm{RM}(4,5)$} \\
\hline$[32,10 ; 4,6]$ & {$[32,16,8] 6 \mathrm{RM}(2,5)$} & {$[32,26,4] \operatorname{RM}(3,5)$} \\
\hline$[32,10 ; 8,12]$ & {$[32,6,16] 12 \mathrm{RM}(1,5)$} & {$[32,16,8] \mathrm{RM}(2,5)$} \\
\hline$[36,20 ; 4,13]$ & {$[36,8,16] \rho \rho \leq 13$} & {$[36,28,4] C_{C}^{\perp}$} \\
\hline$[49,9 ; 20,24]$ & {$[49,1,49] 24$ repetition } & {$[49,10,20][1]$} \\
\hline$[63,6 ; 1,1]$ & {$[63,57,3] 1 \mathrm{BCH}(1)$} & {$[63,63,1]$ full code } \\
\hline$[63,6 ; 3,3]$ & {$[63,51,5] 3 \mathrm{BCH}(2)$} & {$[63,57,3] 1 \mathrm{BCH}(1)$} \\
\hline$[63,6 ; 5,5]$ & {$[63,45,7] 5 \mathrm{BCH}(3)$} & {$[63,51,5] 3 \mathrm{BCH}(2)$} \\
\hline$[63,6 ; 7,7]$ & {$[63,39,9] 7 \mathrm{BCH}(4)$} & {$[63,45,7] 5 \mathrm{BCH}(3)$} \\
\hline$[63,3 ; 9,9]$ & {$[63,36,11] 9 \mathrm{BCH}(5)$} & {$[63,39,9] 7 \mathrm{BCH}(4)$} \\
\hline $\begin{array}{c}{[64,15 ; 4,8]} \\
{[64,15 ; 16,28]}\end{array}$ & \multicolumn{2}{|c|}{$(u, u+v)$ construction } \\
\hline
\end{tabular}

Table 3. Some nested codes for $n \geq 7$. 


\section{Some upper bounds on the nested code dimension}

Lemma 14. For an $[n, K ; d, d]$ nested code, we have

$$
2^{K} \leq\left(\begin{array}{l}
n \\
d
\end{array}\right)+1
$$

Proof. Consider the points of $C_{C}$ and the balls of radius $\rho=d$ around these points. Because $\rho$ is the covering radius of $C_{C}$, these balls cover the space. Since $C_{F}$ has minimum distance $d=\rho$, it can only contain points on the border of these balls, besides the points of $C_{C}$. Hence

$$
\# C_{F} \leq \# C_{C} \cdot\left(\left(\begin{array}{l}
n \\
d
\end{array}\right)+1\right)
$$

and hence

$$
\#\left(C_{F} / C_{C}\right) \leq\left(\left(\begin{array}{l}
n \\
d
\end{array}\right)+1\right)
$$

as required.

We have seen that this bound can be met with equality for $\rho=1$. For $\rho>1$ except $\rho=n=2$ we have inequality; let's see this for $\rho=2$ first.

Proposition 1. For an $[n, K ; 2,2]$ nested code with $n>2$, we have

$$
2^{K}<\left(\begin{array}{l}
n \\
2
\end{array}\right)+1
$$

Proof. Suppose the bound were met with equality. Since $C_{C}$ is a covering code of $\rho=2$, we have

$$
2^{n} \leq 2^{k_{C}}\left(1+\left(\begin{array}{l}
n \\
1
\end{array}\right)+\left(\begin{array}{l}
n \\
2
\end{array}\right)\right) \leq 2^{k_{C}}\left(2^{K}+n\right) \leq 2^{k_{F}}+2^{k_{C}} n .
$$

For all $n>2$, we have

$$
n<1+\left(\begin{array}{l}
n \\
2
\end{array}\right)
$$

which is equal to $2^{K}$ by assumption. This gives

$$
2^{n}<2^{k_{F}+1}
$$

and clearly $n \geq k_{F}$, so we get $n=k_{F}$; but then $d=1<2$, giving a contradiction.

We do have degenerate $[n, 1 ; n, n]$ nested codes for all $n$. They have only the zero word for $C_{C}$, an $[n, 1, n]$ repetition code for $C_{F}$.

Proposition 2. For an $[n, K ; d, d]$ nested code, we have

$$
2^{K} \leq A(n, d, d)+1 .
$$


It is readily seen that this bound is stronger than Lemma 14 when $\rho>2$.

Proof. We start as we did proving Lemma 14 with the balls of radius $\rho$ around the points of $C_{C}$. The border of the ball around $\mathbf{x}$ are the points $\mathbf{x}+\mathbf{y}$ where $\mathbf{y}$ has weight $\rho$. Obeying the distance requirement, the $\mathbf{y}$ that we choose for $C_{F}$ from this ball, will have to form a constant weight code of weight and distance $\rho=d$.

Generalising, we get the following proposition, for which we ommit the proof.

Proposition 3. For an $[n, K ; d, \rho]$ nested code, we have

$$
2^{K} \leq 1+\sum_{w=d}^{\rho} A(n, d, w) .
$$

\section{Some constructions}

Theorem 3. Let $U=U_{F} / U_{C}$ and $V=V_{F} / V_{C}$ be $\left[n, K_{U} ; d_{U}, \rho_{U}\right]$ and $\left[n, K_{V} ; d_{V}, \rho_{V}\right]$ nested codes. Let $U_{i} \circ V_{i}$ denote the $(\mathbf{u}, \mathbf{u}+\mathbf{v})$ composition of $U_{I}$ and $U_{V}$. Then we can form a nested code $C=U \circ V=\left(U_{F} \circ V_{F}\right) /\left(U_{C} \circ U_{F}\right)$, and $C$ is a $\left[2 n, K_{U}+K_{V} ; d, \rho\right]$ nested code with $\rho \leq \rho_{U}+\rho_{V}$ and $d=\min \left\{2 d_{V}, d_{U}\right\}$.

The proof is obvious from fundamental results on the parameters of the component codes.

\section{Acknowledgements}

The authors are grateful for codes contributed by Carl Bracken of Dublin, and for the interest of and discussions with Wolfgang Willems of Magdeburg.

\section{References}

1. Carl Bracken. Private communication. 2004.

2. Gérard Cohen, Iiro Honkala, Simon Litsyn, and Antoine Lobstein. Covering codes, volume 54 of North-Holland Mathematical Library. North-Holland Publishing Co., Amsterdam, 1997.

3. Max H. M. Costa. Writing on dirty paper. IEEE Trans. Inform. Theory, 29(3):439$441,1983$.

4. Frank Kschischang and David Tse, editors. Proc. IEEE Intern. Symp. Inform. Theory, pages 533-536. June 2004. A four-talk session on dirty paper coding.

5. M.L. Miller, G.J. Doerr, and I.J. Cox. Applying informed coding and embedding to design a robust high-capacity watermark. IEEE Transactions on Image Processing, 13(6):792-807, June 2004. 